\title{
Case Series and Review of the Literature
}

\section{Delivery of Cancer Care at Home during the First COVID-19 Pandemic in Italy: A Case Series Study (DomOnCOVID Project)}

\author{
Rodolfo Passalacqua $^{I^{*}}$, Margherita Ratti ${ }^{1}$, Maria Bonomi ${ }^{1}$, Giulia Grizzi ${ }^{1}$, Federica Negri ${ }^{1}$, Francesco Crea ${ }^{2}$, Mariaolga \\ Giganti $^{1}$, Bruno Perrucci ${ }^{1}$, Matteo Brighenti ${ }^{1}$, Stefano Panni, Maddalena Donini ${ }^{1}$, Alessandra Curti, Daniele Spada ${ }^{I}$, \\ Roberta Marchi ${ }^{1}$, Gianvito Donati ${ }^{1}$, Jessica Saleri ${ }^{1}$, Morena Nazzari ${ }^{1}$, Giovanna Pogliacomi ${ }^{1}$, Francesca Diodati ${ }^{3}$ and \\ Caterina Caminiti ${ }^{3}$
}

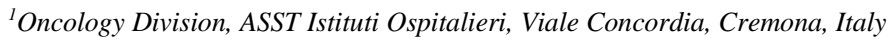

${ }^{2}$ General Practitioner, ATS Val Padana, Distretto di Cremona, Via Miglioli, Soresina, Cremona, Italy

${ }^{3}$ Research and Innovation Unit, University Hospital of Parma, Via Gramsci, Parma, Italy

\section{ART ICLE INFO}

Article history:

Received: 26 May, 2021

Accepted: 9 June, 2021

Published: 21 June, 2021

Keywords:

Home care

COVID-19

cancer

telemedicine

continuity of care

\begin{abstract}
A B S T R AC T
Background: Cancer represents a major risk factor for COVID-19 poor outcomes. During the crucial phase of the pandemic, we launched a home care project, called DomOnCOVID, aiming to provide care to patients in their own homes, enabling to keep immunocompromised individuals away from health care facilities, decrease hospital use, and strengthen hospital capacity for subjects with COVID-19 and other conditions. This paper describes this intervention in terms of feasibility and clinical outcomes.

Methods: This is a descriptive study of cancer patients with confirmed or suspected COVID-19 infection assisted at home in the Italian Province of Cremona during the pandemic's first peak. We devised an organizational home care system which included a medical and nursing team equipped with a car for home visits, and a nurse manager who screened patient calls requesting inclusion in the project. The team administered oral drugs at home (chemotherapy, TKis, etc.) and was equipped with all necessary tools to conduct examinations, check vital signs, take blood samples, and nasopharyngeal swabs for COVID-19 testing.

Results: From March 23rd to May 15th 2020, 71 cancer patients were assisted at home (181 visits, mean 2.5, SD 1.6 range 1-7). All had symptoms that could be traced back to COVID infection, but only 26/71 (37\%) were found to be COVID+; 19/26 (73\%) had mild symptoms, while 7 with severe symptoms were hospitalized and 2 died for COVID-19. The remaining patients recovered. 43/71 (60\%) received at home oral or subcutaneous drugs and no particular problems or toxicity were observed. 16/28 (57\%) of individuals living with COVID+ patients were found to be COVID+, while none of the non-cohabiting were COVID+. Conclusion: Delivery of cancer care at home is feasible and may be particularly useful not only during health crises but also after the epidemic in order to reduce hospital access, patient and care-giver travel and improve their quality of life. Further implementation studies on home-based care in oncology are warranted.
\end{abstract}

\section{Introduction}

The global pandemic of the coronavirus disease 2019 (COVID-19) caused by the novel severe acute respiratory syndrome coronavirus 2 (SARS-CoV-2) has had a major impact on healthcare delivery systems [1]. Hospitals and community services were overwhelmed by the rapidly increasing number of affected patients requiring urgent care, and by the need to limit disease spread and rapidly identify and isolate new cases. In response to this unprecedented crisis, many countries, including Italy, adopted new organizational models that included ward closure and repurposing with cessation or delay of elective procedures [2, 3].

During epidemics, home-based care can help manage high-risk patients, moderate the risk of exposure for healthcare workers, and mitigate 
community transmission of the virus by keeping people at home and away from hospitals, which are themselves potential sources of infection [4]. This is particularly true for COVID-19 patients with underlying conditions or risk factors which place them at high-risk for poor outcomes. In fact, patterns of hospital admission in various countries show that patients admitted to the Intensive Care Unit (ICU) or regular floor for COVID-19 are more likely to have at least one underlying health condition or risk factor (78\% in ICU, 71\% on regular floor) [4]. Cancer represents a major risk factor for COVID-19 poor outcomes. In a systematic review, comparative analysis between COVID patients with and without cancer showed that the former had a higher risk of mortality (odds ratio $[\mathrm{OR}]=3.23,95 \% \mathrm{CI}$ : $1.71-6.13$ ), severe/critical disease $(\mathrm{OR}=3.91,95 \% \mathrm{CI}: 2.70-5.67)$, ICU admission $(\mathrm{OR}=3.10,95 \% \mathrm{CI}$ : 1.85-5.17), and mechanical ventilation ( $\mathrm{OR}=4.86,95 \% \mathrm{CI}: 1.27-18.65)$ [5]. These results indicate that individuals with cancer need diligent preventive care measures and aggressive surveillance for earlier detection of COVID-19, given their higher risk of infection-related complications.

Italy was the first European country hit by the COVID-19 epidemic. Since the first confirmed case identified on February 20th, 2020 in Lombardy (the region of Milan) in Northern Italy, the outbreak quickly spread nationwide, particularly in the North, severely affecting the National Health Service [6]. The town of Cremona, in Lombardy, was severely affected. During the first 8 weeks the town's hospital, a 500bed facility, evaluated 1706 patients with suspected COVID-19 in the Emergency Department, of whom 1542 were hospitalized [7]. In midMarch 2020, during the crucial phase of the pandemic, we launched a home care project, called DomOnCOVID, with the aim of avoiding hospital admissions of cancer patients and their families, by following patients with mild/moderate symptoms at home and admitting only those with severe symptoms who needed invasive respiratory support.

This paper aims to describe this intervention in terms of feasibility and clinical outcomes. The results of this pilot study can help decide whether the programme could be routinely implemented in COVID emergency and in post-emergency conditions.

\section{Material and Methods}

This study describes a consecutive case series of cancer patients receiving home care in the town of Cremona and its province at the first pandemic's peak, when the hospital was almost completely occupied by COVID-19 patients.

The main objective of the study was to verify the feasibility of home care on a consecutive series of COVID-19+ cancer patients or subjects with suspected symptoms of COVID-19 infection who did not have clinical and instrumental criteria that required hospitalization. For this purpose, the following events were measured on the total number of patients cared for at home:
i. Hospitalization for COVID-19 complications.
ii. ICU admission or use of mechanical ventilation.
iii. Cancer treatment interruption.

The project also included COVID-19 screening for patient cohabitants and close contacts. Secondary objective was therefore to describe screening outcome for these subjects.

\section{Study Population}

All patients had a confirmed cancer diagnosis, and an established diagnosis of COVID-19 infection or suspected symptoms of COVID-19 infection: fever, cough, mucositis, loss of smell, etc. Patients exhibiting a clinical situation and/or symptoms requiring immediate hospitalization were excluded.

\section{Interventions}

Key element of the study is the creation of the Home Care Management Team (HCMT), composed of clinicians (medical oncologists and oncology nurses) from the Oncology Division of Cremona Hospital, and a manager nurse, who screened patient calls received at a dedicated number requesting to be included in the project, and performed telephone triage to determine the priority of home interventions. Home care was provided from Monday to Saturday, from 8 AM to 5 PM. After $5 \mathrm{pm}$ and on Sundays, patients could call the Oncology Department for a telephone consultation and were advised to directly refer to the Hospital Emergency Room in case of rapid worsening of conditions.

The hospital management provided the HCMT with a car and the necessary equipment to guarantee safety of health care professionals during home visits. The volunteer association MEDeA (Medicine and Art, OdV, Cremona) supplied two portable oxygen concentrators that could be left at the patient's home if required. The HCMT worked in strict collaboration with health care professionals in the community, including general practitioners. All patients were tested for COVID-19 with at least one nasopharyngeal swab (NPS). The same procedure was performed on their cohabitants (both symptomatic and nonsymptomatic) and on non-cohabiting close contacts. The HCMT conducted blood tests and medical examinations (measurement of blood pressure, oxygen saturation, heart and respiratory rate, electrocardiogram). All screened individuals were advised to follow quarantine procedures and were provided with an information leaflet indicating the prophylactic measures they should take to avoid infecting contacts. Oral/infusion treatments were administered, including antiviral drugs according to FADOI (Federation of Associations of Hospital Internists) guidelines [8]. Patients were followed up until resolution of symptoms or, in case of NPS positivity, until two consecutives negative NPSs were obtained (48 hours one apart from the other).

\section{Results}

From March 23rd to May 15th 2020, 71 cancer patients were assisted at home, with a total of 181 home visits (mean 2.5, SD 1.6, range 1-7). All patients had at least one moderate or severe symptom which, theoretically, could be related to COVID infection. Patient characteristics are shown in (Table 1). Median age was 71 years (IQR 66-85), the majority was male $(53 / 71,75 \%)$, Performance Status (PS) was $0-1$ in $43 / 71(61 \%)$ patients, 53/71 (75\%) had metastatic cancer and approximately half of them was receiving chemotherapy when home care began. 
Table 1: Patient Characteristics.

\begin{tabular}{|l|l|}
\hline Characteristic & Total (n=71) \\
\hline Age, median (IQR) & $71(66-85)$ \\
\hline Sex & \\
\hline Male & $53(75 \%)$ \\
\hline Female & $18(25 \%)$ \\
\hline Performance Status (ECOG) & \\
\hline $2-3$ & $43(61 \%)$ \\
\hline Primary tumor & $28(39 \%)$ \\
\hline Lung & \\
\hline Colon & $16(23 \%)$ \\
\hline Prostate & $9(13 \%)$ \\
\hline Urothelial & $5(7 \%)$ \\
\hline Liver & $4(6 \%)$ \\
\hline Gynaecologic & $4(6 \%)$ \\
\hline Breast & $4(6 \%)$ \\
\hline Other Gastrointestinal & $2(3 \%)$ \\
\hline Other Genitourinary & $17(24 \%)$ \\
\hline Others & $3(4 \%)$ \\
\hline Metastasis & $7(10 \%)$ \\
\hline Yes & \\
\hline No & $53(75 \%)$ \\
\hline Ongoing treatment & $18(25 \%)$ \\
\hline Chemotherapy & \\
\hline Chemotherapy + Immunotherapy & $22(31 \%)$ \\
\hline Immunotherapy only & $7(10 \%)$ \\
\hline Target therapy & $9(13 \%)$ \\
\hline Supportive care & $13(18 \%)$ \\
\hline Others or none & $8(11 \%)$ \\
\hline & $12(17 \%)$ \\
\hline
\end{tabular}

All patients were tested at home with NPS (Table 2). 26/71 (37\%) were found to be COVID-19 positive, of whom $6 / 26$ (23\%) had already been diagnosed with COVID-19 during a hospital visit. Among COVID-19 positive patients, $19 / 26(73 \%)$ exhibited mild symptoms (fever $<38^{\circ} \mathrm{C}$, cough, olfactory alterations, mild dyspnea with oxygen saturation $>93 \%$ in room air), while $7 / 26$ (27\%) had pneumonia with stable vital parameters. During subsequent home visits, 6/7 (86\%) patients experienced worsening of respiratory symptoms and were immediately hospitalized, 1/7 (14\%) patient initially refused hospitalization until his condition worsened and he was admitted to the Pneumology Department, where he died 3 days later. Of the 6 hospitalized individuals, 3 improved and were subsequently discharged and followed at home, while 3 died, 1 from COVID-19 pneumonia and 2 from tumor progression. None of the 7 hospitalized patients was admitted to ICU or received invasive mechanical ventilation.

45/71 (63\%) patients were found to be COVID-19 negative. The vast majority $(39 / 45,87 \%)$ had mild symptoms, and no one was hospitalized or died during the observation period. All COVID-negative patients who were undergoing anticancer treatment received their oral or subcutaneous or IV therapy at home and were able to continue treatment without returning to hospital. 64 persons were in contact with COVID19+. 28/64 (44\%) lived with the patient while 36/64 (56\%) were close contacts, i.e., visited the patient at least once a day. Interestingly, of the 28 cohabiting individuals, 16 (57\%) were found to be COVID-19+ by NPS testing, none of whom knew they were carriers. 2 of them were hospitalized, and eventually died of complications of COVID-19. None of the 36 non-cohabiting close contacts tested positive for COVID-19. No health professional was infected with COVID-19 during home care activities.

Regarding the costs of this project, we had not planned a prospective cost analysis, however, we can make an estimate of main health care expenses incurred assuming one year of activity and 200 patients followed at home. These are shown in (Table 3) and mainly concern personnel expenses, the car and other equipment, in addition to personal protective equipment.

Table 2: Results of COVID-19 screening of patients and their cohabiting and non-cohabiting family members.

\begin{tabular}{|l|l|l|}
\hline Individuals screened at Home & COVID-19 Positive \# $(\%)$ & COVID-19 Negative \# (\%) \\
\hline Patients screened $(\mathrm{n}=71)$ & $26(37 \%)$ & $45(63 \%)$ \\
\hline Mild symptoms & $19(73 \%)$ & $39(87 \%)$ \\
\hline Severe symptoms & $7(27 \%)$ & $6(13 \%)$ \\
\hline Hospitalized & $7(27 \%)$ & $0(0 \%)$ \\
\hline Deaths & $4(15 \%)$ & $0(0 \%)$ \\
\hline Contacts screened $(\mathrm{n}=64)$ & & \\
\hline Cohabiting*(n=28) & $16(57 \%)$ & $12(43 \%)$ \\
\hline Hospitalized & $2(13 \%)$ & 0 \\
\hline Deaths & $2(13 \%)$ & 0 \\
\hline Close contacts**, no cohabiting $(\mathrm{n}=36)$ & $0(0 \%)$ & $36(100 \%)$ \\
\hline
\end{tabular}

*Cohabiting: person living with patient.

**Close contacts: who visited the patient at least once a day.

Table 3: Estimated costs incurred assuming one year of activity in which about 200 patients receive oncological home care. Drug costs are not included.

\begin{tabular}{|l|l|}
\hline Cost items & Amount in Euro for 1 year \\
\hline Staff & \\
\hline 2 full-time nurses & 80,00 \\
\hline
\end{tabular}




\begin{tabular}{|l|l|}
\hline 1 part-time physician & 30,00 \\
\hline Car (including insurance, gas and maintenance costs) & 10,00 \\
\hline PPE (Personal Protective Elements) & 15000,00 \\
\hline Electrocardiograph $\mathrm{n}=1$ & 700,00 \\
\hline Oxygen concentrator $\mathrm{n}=2$ & 1800,00 \\
\hline Pulse oximeter $\mathrm{n}=2$ & 200,00 \\
\hline Sphygmomanometer $\mathrm{n}=2$ & 200,00 \\
\hline Other expenses & 5000,00 \\
\hline Total expenses for one year & 142,900 \\
\hline
\end{tabular}

\section{Discussion}

The COVID-19 pandemic is putting a strain on health systems around the world. The health crisis has changed the way care is provided and has forced doctors to make difficult decisions about which types and components of care should be maintained. By limiting the number of unnecessary hospital accesses of patients and caregivers, home care may be useful to contain COVID-19 spread. Moreover, it may reduce the distress of more fragile individuals, such as people with cancer, by guaranteeing essential care, close monitoring and timely intervention, especially in case of COVID-19 positivity.

This study describes the feasibility of an innovative model based on home care for cancer patients with confirmed or suspected COVID-19+ and mild symptoms. In this model, patients are assisted at home by the same health care professionals who care for them at the hospital, which has multiple advantages. Firstly, patients may feel more comfortable being handled by clinicians who already know them. Furthermore, hospital staff can ensure direct patient referral to the ward in case admission becomes necessary, thus avoiding access to the chaotic and overcrowded Emergency Department. Over approximately 40 days, at the peak of the epidemic's first wave, our team was able to perform 181 home visits, about $4 / 5$ a day, a considerable effort in the light of the devastating health crisis. The findings described in this paper seem to suggest a favourable impact of our intervention on the negative effects of the pandemic for cancer patients, although the lack of a control group does not enable us to draw conclusions on effectiveness.

The literature indicates cancer as a major risk factor for COVID-19 poor outcomes, although published studies on this aspect differ in cancer types, stage, performance status, comorbidities and other confounding factors of included patients [9-11]. For example, reported mortality rates range from $13 \%$ to $33 \%[9,11]$. According to a systematic review and meta-analysis of 22 studies (1018 cancer patients), mortality in cancer patients with COVID-19 infection was $21.1 \%$ (95\% CI: 14.7-27.6), ICU admission rate was $14.5 \%$ (95\% CI: 8.5-20.4), and mechanical ventilation rate was $11.7 \%$ (95\% CI:5.5-18) [5]. The findings of our study appear to be more favourable (overall mortality rate for COVID19 was $15 \%$, no patient was admitted to the ICU or was intubated), however, we cannot know whether populations are comparable, as the systematic review does not indicate COVID-19 severity of included patients [5].

The DomOnCOVID project extended COVID-19 screening to symptomatic and asymptomatic close contacts (mostly cohabiting family members). In our experience, the probability to identify asymptomatic positive subjects among persons living with a COVID-19+ was more than $50 \%$. Contact tracing and screening of asymptomatic individuals are essential measures to contain an outbreak [12]. Contact tracing of COVID-19+ patients, compared to symptomatic surveillance, reduces the time in which contacts are contagious in the community [13]. This is particularly important for crowded indoor environments such as households with sustained close contacts. According to a systematic review and metanalysis on household transmission of SARS-CoV-2, secondary attack rate (number of new infections among contacts over the total number of contacts within the household or family) was 17\% [14]. In our experience, 16/64 (25\%) contacts tested positive for COVID-19. The higher secondary attack rate we observed may be due to the precise classification of "close contact" in our study (person visiting the patient at least once a day); in the review close contact definitions varied by study, and family members were included even when living outside the patient's household [14]. Furthermore, the review does not concern cancer patients, a population likely to have greater need for close caregiver assistance, which may increase contact risk of infection.

A recent experience from the University of Pennsylvania has shown that, for appropriate cancer drugs and patient populations, home administration of cancer drugs can replace inpatient or outpatient administration. Furthermore, they found that during and after the COVID pandemic there was a $700 \%$ increase in the number of patients participating in the cancer care at home programme and an increase of the number of cancer drugs provided at home [15]. This study has several limitations. Firstly, the lack of a control group did not allow to assess the real effects of the intervention, only enabling the description of findings and comparisons with highly variable literature data. Although the randomized trial is the gold standard, randomizing patients to the intervention was deemed unethical. Moreover, recruiting an additional cancer center as control was considered impossible in the light of the emergency. Secondly, sample size was not defined a priori, but was determined by the epidemic's trend, whereby the number of COVID-19 patients drastically decreased after May $15^{\text {th }}$. Thirdly, this experience was limited to citizens residing in the Cremona area and was conducted in only one hospital, which does not ensure generalizability of the model to other settings.

Building upon the results of DomOnCOVID, we have planned the implementation of a home-based care model to patients receiving oral treatments, regardless of COVID-19 symptoms. This new project, called OncoHome, involving three cancer centers in the Lombardy Region of Italy, will provide further data on the feasibility and cost-effectiveness of home care. 


\section{Conclusion}

This model is feasible and may be particularly useful during health crises such as the current COVID-19 epidemic. It will be important to determine if home-care programmes of this kind may be beneficial also beyond emergencies. To this end, further implementation studies on home-based care in oncology after the epidemic are warranted.

\section{Acknowledgement}

We would like to thank:

i. The Health Department of the ASST of Cremona for their unconditional support to the project

ii. The Italian Association of Medical Oncology (AIOM) and the Medical Order of Cremona for their patronage for this work.

\section{Author Contributions}

RP, MR, conceived the study. CC contributed to study design, analyses of the data and advice on statistical methods. MR, GG, MG, BP, MB, SP, MD, AC, DS, FD supervised the study and provided comments on the manuscript draft. FN, FC, RM, GP, GD, JS, MN, Provision of study materials or patients, collection and assembly of data. RP, MB, CC prepared the draft of the manuscript, tables. All authors read and approved the final manuscript.

\section{Funding}

This work was supported by:

i. The Azienda Socio Sanitaria Territoriale (ASST) of Cremona;

ii. The non-profit organization MEDeA (Medicine and Art, OdV), Cremona;

iii. The Association "Uniti per la Provincia di Cremona".

\section{Availability of Data and Materials}

The datasets used and analysed during the current study are available from the corresponding author on reasonable request.

\section{Ethics Approval and Consent to Participate}

This was an organizational intervention conducted with the hospital management's support in emergency conditions. The project did not require formal ethical evaluation and approval, but the Ethics Committee was informed about the project. All patients had given written consent at the time of anticancer treatment initiation at the hospital. Since home care was conceived as a way to ensure assistance during the pandemic, informed consent to the use of data for research purposes was collected following a deferred-consent procedure (as recommended during emergencies), at home or at the patient's first visit to the Oncology Department.

\section{Consent for Publication}

Not applicable.

\section{Competing Interests}

Rodolfo Passalacqua has participated in the last 2 years in advisory boards or as a speaker for: Astellas, BMS, Ipsen, Janssen, SanofiAventis, Roche, MSD. Institutional funding for research projects from: Amgen, Pierre-Fabre, Astra Zeneca, Novartis.

\section{Conflicts of Interest}

None.

\section{Trial Registration}

Not Applicable.

\section{Supplementary Information}

None.

\author{
Abbreviations \\ COVID-19: Coronavirus Disease 2019 \\ FADOI: Federazione delle Associazioni Dirigenti Ospedalieri Internisti \\ (Federation of Associations of Hospital Internists) \\ HTMT: Home Care Management Team \\ ICU: Intensive Care Unit \\ MEDeA: Medicine and Art \\ NPS: Nasopharyngeal Swab \\ SARS-CoV-2: Severe Acute Respiratory Syndrome Coronavirus 2
}

\section{REFERENCES}

1. Wang C, Horby PW, Hayden FG, Gao GF (2020) A novel coronavirus outbreak of global health concern. Lancet 395: 470-473. [Crossref]

2. Remuzzi A, Remuzzi G (2020) COVID-19 and Italy: what next? Lancet 395: 1225-1228. [Crossref]

3. Sorbello M, El Boghdadly K, Di Giacinto I, Cataldo R, Esposito C et al. (2020) The Italian coronavirus disease 2019 outbreak: recommendations from clinical practice. Anaesthesia 75: 724-732. [Crossref]

4. Medina M, Babiuch C, Card M, Gavrilescu R, Zafirau W et al. (2020) Home monitoring for COVID-19. Cleve Clin J Med. [Crossref]

5. ElGohary GM, Hashmi S, Styczynski J, Kharfan Dabaja MA, Alblooshi RM et al. (2020) The risk and prognosis of COVID-19 infection in cancer patients: A systematic review and meta-analysis. Hematol Oncol Stem Cell Ther. [Crossref]

6. Reno C, Lenzi J, Navarra A, Barelli E, Gori D et al. (2020) Forecasting COVID-19-Associated Hospitalizations under Different Levels of Social Distancing in Lombardy and Emilia-Romagna, Northern Italy: Results from an Extended SEIR Compartmental Model. J Clin Med 9: 1492. [Crossref]

7. Pan A, Giorgi Pierfranceschi M, Bosio G, Cammelli L, Canino R et al. (2020) Suggestions from Cremona, Italy: 2 months into the pandemic at the frontline of COVID-19 in Europe. Clin Microbiol Infect 26: 1127-1129. [Crossref] 
8. COVID-19 (2020) Guida clinico-pratica FADOI. FADOI Società Scientifica di Medicina Interna.

9. Kuderer NM, Choueiri TK, Shah DP, Shyr Y, Rubinstein SM et al. (2020) Clinical impact of COVID-19 on patients with cancer (CCC19): a cohort study. Lancet 395: 1907-1918. [Crossref]

10. Lee LYW, Cazier JB, Starkey T, Briggs SEW, Arnold R et al. (2020) COVID-19 prevalence and mortality in patients with cancer and the effect of primary tumour subtype and patient demographics: a prospective cohort study. Lancet Oncol 21: 1309-1316. [Crossref]

11. Garassino MC, Whisenant JG, Huang LC, Trama A, Torri V et al. (2020) COVID-19 in patients with thoracic malignancies (TERAVOLT): first results of an international, registry-based, cohort study. Lancet Oncol 21: 914-922. [Crossref]
12. Keeling MJ, Hollingsworth TD, Read JM (2020) Efficacy of contact tracing for the containment of the 2019 novel coronavirus (COVID-19). J Epidemiol Community Health 74: 861-866. [Crossref]

13. Bi Q, Wu Y, Mei S, Ye C, Zou X et al. (2020) Epidemiology and transmission of COVID-19 in 391 cases and 1286 of their close contacts in Shenzhen, China: a retrospective cohort study. Lancet Infect Dis 20: 911-919. [Crossref]

14. Madewell ZJ, Yang Y, Longini IM Jr, Halloran ME, Dean NE et al. (2020) Household Transmission of SARS-CoV-2: A Systematic Review and Meta-analysis. JAMA Netw Open 3: e2031756. [Crossref]

15. Laughlin AI, Begley M, Delaney T, Zinck L, Schuchter LM et al. (2020) Accelerating the Delivery of Cancer Care at Home During the Covid-19 Pandemic. NEJM Catalyst. 